\title{
Simulation and Proposed Handover Alert Algorithm for Mobile Communication Networks
}

\author{
$\underline{\text { doi:10.3991/ijim.v3s2.838 }}$ \\ Muzhir Al-Ani ${ }^{1}$ and Wael Al-Sawalmeh ${ }^{2}$ \\ ${ }^{1}$ Amman Arab University, Amman, Jordan \\ ${ }^{2}$ Philadelphia University, Amman, Jordan
}

\begin{abstract}
This paper deals with a novel approach to realize the handover process of a mobile systems network. It concentrates on the existing challenges of Global System for Mobile communication (GSM) networks and how to overcome these challenges. The proposed algorithm extracts the received signal information features in order to track the significant coverage cell. The presented algorithm distinguishes between real problems and false alarms. One of the important contributions of the algorithm is how it predicts the adequate signal of the effective coverage cell.
\end{abstract}

Index Terms-Handover, Coverage Area Measurements, Mobile Positioning \& Digital Cellular System

\section{INTRODUCTION}

During the early 1980 s, analog cellular telephone systems were experiencing rapid growth in Europe, particularly in Scandinavia and the United Kingdom, but also in France and Germany. Each country developed its own system, which was incompatible with everyone else's in equipment and operation.

In 1989, GSM responsibility was transferred to the European Telecommunication Standards Institute (ETSI), and phase I of the GSM specifications were published in 1990. Commercial service was started in mid-1991, and by 1993 there were 36 GSM networks in 22 countries. Although standardized in Europe, GSM is not only a European standard. Over 200 GSM networks (including DCS1800 and PCS1900) are operational in 110 countries around the world. In the beginning of 1994, there were 1.3 million subscribers worldwide, which had grown to more than 55 million by October 1997. With North America making a delayed entry into the GSM field with a derivative of GSM called PCS1900, GSM systems exist on every continent, and the acronym GSM now aptly stands for Global System for Mobile communications.

The developers of GSM choose an unproven digital system, as opposed to the then-standard analog cellular systems like AMPS in the United States and TACS in the United Kingdom. They had faith that advancements in compression algorithms and digital signal processors would allow the fulfillment of the original criteria and the continual improvement of the system in terms of quality and cost.

The revolution of the telecommunication technology opens new doors in many countries all over the world to start development and investigation over mobile system fields starting from the analog systems and then to digital systems that allow the subscriber mobility and comfort.
The cellular system as implemented in most countries demonstrates that the world is a small village. On the other hand, a substantial growth in the number of subscribers makes it necessary to evaluate large and extended networks to overcome the growth of mobile networks [1], thus GSM becomes the most popular network. Nowadays there are many different and potential types of GSM with different features and applications. The popularity of mobile phones and the number of mobile devices users is continuously increasing, so manufacturers are continually trying to introduce new features and services to attract new customers [2].

GSM and $2 \mathrm{G}$ networks have been started in many Arab countries during 1990s, while the transition from $2 \mathrm{G}$ to $3 \mathrm{G}$ started at the beginning of 2000s. Due to some financial problems, some countries have not evolved to $2 \mathrm{G}$ or $3 \mathrm{G}$ technologies. Therefore, our work is concentrated on improving the GSM network performance, since it is still widely used.

\section{Digital COMMUNiCATION SySTEM}

The usage of mobile communication systems keeps expanding, leading to large number of studies related to GSM services, techniques and algorithm improvement. A fundamental goal of mobile telephone network operators is to provide seamless service for their subscribers [3] \& [4].

The report of the GSM Association in 2004 indicates that the number of GSM networks exceeds 500 in more than 180 countries worldwide; the Latina American and Asian Pacific regions have the largest share of the highest rates of growth in both number of subscribers and number of networks lunched [5].

As in [6], developing and proposing a new algorithm for receiving GSM broadcasted data is important because it allows subscribers to obtain the identification parameters and carry out an analysis of mutual interference effects.

Cellular Systems are moving from $2 \mathrm{G}$ to $3 \mathrm{G}$, while $2 \mathrm{G}$ already brings high speed data transmission enabling wideband multimedia applications. The next generation of cellular systems will be increasingly similar to a data communication system, which not only transfers voice and multimedia, but will also be integrated with WLAN to access Internet [7].

Great effort has been devoted towards the design and development of mobile architectures and multiservice networks. High performance modeling and evaluation of General Packet Radio Service (GPRS) are adapted via 
GSM cell involving both voice and multiple class data services under a complete partitioning scheme [8].

GPRS provides packet switched data transfer to efficiently utilize the radio resources. GPRS is considered the main development step of GSM networks toward the next generation of mobile communication system like UMTS. GPRS allows a single mobile station to transmit data using multiple time slots to increase the transmission rate [9].

Existing GSM/GPRS base station site utilization can be sped up $3 \mathrm{G}$ wide band code division multiple access (WCDMA) deployment. It is possible to provide full coverage even for bit rates higher than 144 or $384 \mathrm{kbps}$ when 3G WCDMA use GSM 1800 sites [10].

As operators continue to roll out networks, it is obvious that providing the depth of coverage equal to the massmarket population coverage achieved with GSM 900/1800 will not be possible for many years. Early release of UMTS enables circuit-switched users to access similar services via GSM or UMTS [11].

Detecting and explaining fully states in complex telecommunications systems such as GSM networks are mentioned as challenging tasks. Mobile networks are hierarchical cell-based systems with complex dynamics influenced by the stochastic user demand. The network operating characteristic such as hand over algorithms, carrier frequency and the non-stationary influence of the environment [12] are considered as main areas of this field.

\section{Digital Celluler System}

Digital cellular systems are implemented in numerous countries all over the world. This system consist of three main parts: mobile switching center (MSC), base station system (BSS) and mobile station (MS). Each division is divided into other entities. The overall system is connected to the normal public service telephone network (PSTN) as shown in Figure 1 [13]. In cellular systems, the coverage area of an operator is divided into cells. These cells are normally hexagonally shaped, but in practice, because of the influence of the terrain, the shape is irregular as shown in Figure 2. The size of cells varies from a few hundred meters to many tens of kilometers, depending on the coverage area and the number of subscribers and transmitting power [14].Each base station (BS) is allocated to a different carrier frequency and each cell has a usable bandwidth associated with this carrier. The cellular radio permits the use of a limited part of the radio spectrum, so the available number of carrier frequencies is limited [15].

Therefore, to provide sufficient channels for more subscribers, it is necessary to re-use the available frequencies many times, which creates possible interference between cells carrying the same frequencies. To avoid interference it is preferable to isolate the cells by employing different frequencies [1].

The cells with different carrier frequencies form a section which identifies the re-use distance, which can depend on many factors, including the number of co-channel cells in the vicinity of the center cell and the geography of the terrain and the transmitted power within each cell [16].

Mobile network providers installing thousands of base stations in each country implies using small cells, although it is more expensive than using big cells.

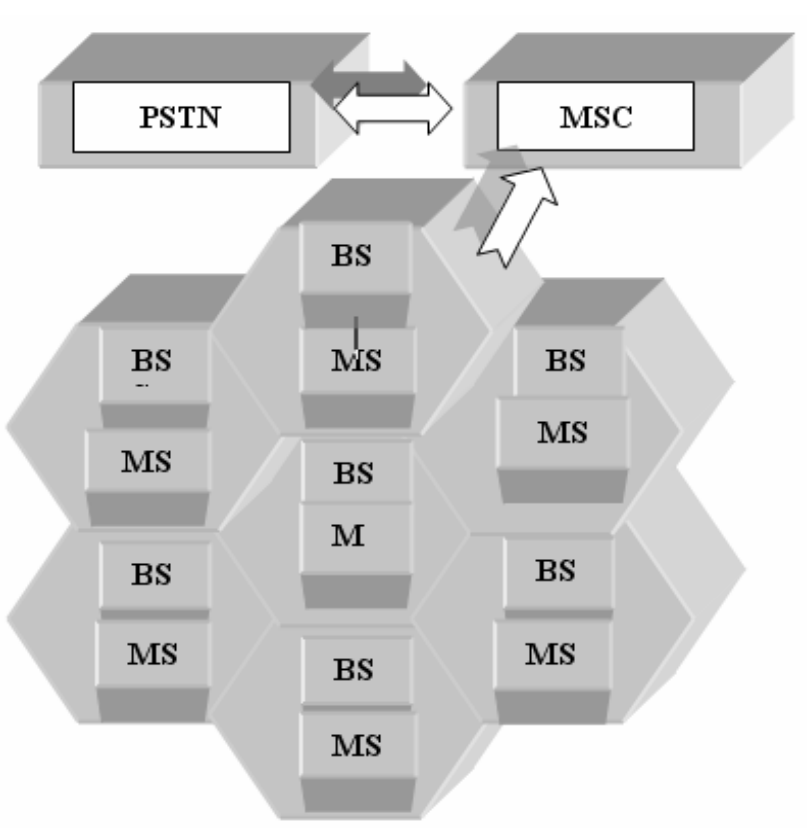

Figure 1. Main parts of Cellular System

The advantages of cellular systems with small cells are:

- Higher capacity, they are limited to fewer possible users per $\mathrm{km}^{2}$.

- Less transmission power, a receiver near the base station requires less power.

- Only local interference occurs.

- Robustness, if one cell fails, the problem occurs in small area.

Small cells also have disadvantages:

- They need a complex infrastructure to connect all base stations.

- They require a fast handover because of mobility between cells.

- Frequencies have to be distributed carefully to avoid interference between transmitters.

\section{GSM SYSTEM ARCHITECTURE}

GSM became popular rapidly because of the quality improvement and its use of international standards. The GSM communication network is divided into three main groups; the mobile station (MS), the base station subsystem (BSS) and the network subsystem (NS), which is illustrated in Figure 3.

The MS or mobile equipment (ME) includes the subscriber identity module (SIM). Each SIM card has an identification number called international mobile subscriber identity (IMSI). Each MS is assigned to a hardware identification called international mobile equipment identity (IMEI). Besides providing transmission and reception of voice and data, the mobile performs other tasks such as authentication, handover encoding and channel encoding [17] \& [18].

The BSS consists of the base station controller (BSC) and the base transceiver station (BTS). The BTS is used to connect the mobiles to a cellular network.

Their tasks include channel coding/decoding and encryption/decryption. A BTS is comprised of radio trans- 


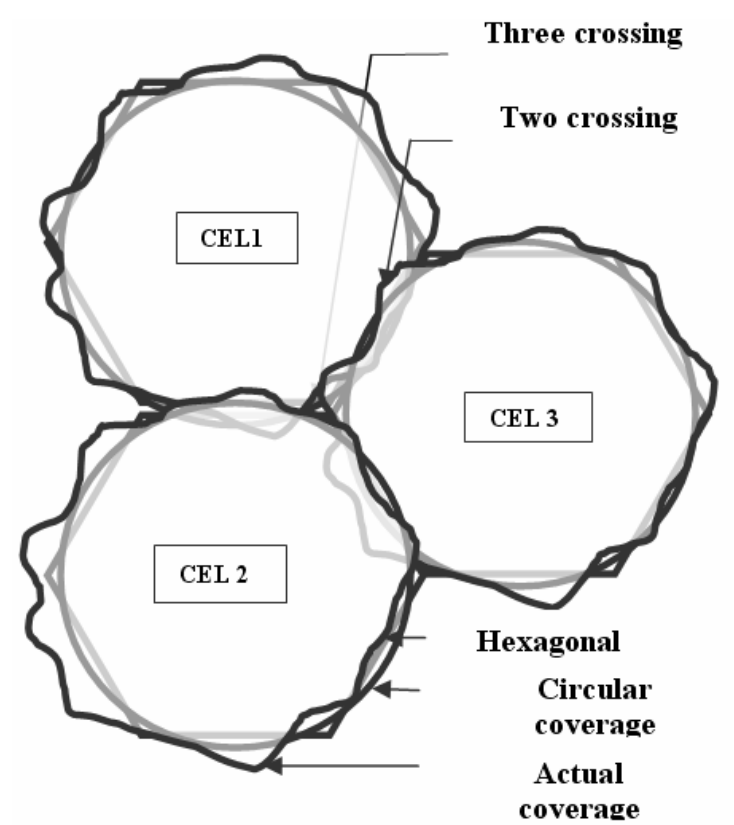

Figure 2. Effects of crossing on the coverage

mitters and receivers, antennas and an interface to the PCM facility ...etc. A group of BTS are connected to a particular BSC. The main function of the BSC is call maintenance. The MS sends a report of their received signal strength to the BSC, so the BSC decides to initiate hand over to other cell [19].

The network subsystem includes the mobile switching center (MSC), the home location register (HLR), the visitor location register (VLR), the authentication center (AUC) and the equipment identity register (EIR). The main functions of MSC are registration, authentication, location updating, handovers and call routing to a roaming subscriber. MSC also has a gateway function for communication with other networks. HLR is a data base used for management of mobile subscribers; it stores the IMSI, mobile station ISDN number (MSISDN) and current visitor location register (VLR) address.

VLR contains the current location of the MS and selected administrative information from the HLR, necessary for call control and the provision of subscriber services, for each mobile currently located in the geographical area controlled by the VLR, which is connected to one MSC. AUC is a protected database that holds a copy of the secret key stored in each subscriber's SIM card which is used for authentication and encryption. The EIR is the database that contains a list of all valid mobile station equipment within the network, where each mobile station is identified by its IMEI. The operation and management center $(\mathrm{OMC})$ is a management system which maintains the operation of the GSM network. The OMC is responsible for controlling and maintaining the MSC, BSC and BTS.

\section{GeOgraphicAl AREAS OF GSM Network}

In order to work properly, a cellular system must verify the following main conditions:

- The power level of a transmitter within a single cell must be limited in order to reduce the interference with the transmitters of neighboring cells. The interference will not produce any damage to the system if a distance of about 2.5 to 3 times the diameter of a cell is reserved between transmitters.

- Neighboring cells can not share the same channels. In order to reduce the interference, the frequencies must be reused only within a certain pattern.

- In GSM, five main functions must be defined: Transmission, Radio Resources management (RR), Mobility Management (MM), Communication Management (CM), Operation, and Administration and Maintenance (OAM).

The geographical area of a GSM network is illustrated in Figure 4. In a GSM system, a cell is identified by its Cell Global Identity number (CGI), corresponding to the radio coverage of a base transceiver station. A Location Area (LA), identified by its Location Area Identity (LAI) number, is a group of cells served by a single MSC/VLR. A group of location areas under the control of the same MSC/VLR defines the MSC/VLR area. A Public Land Mobile Network (PLMN) is the area served by one network operator.

$$
\begin{array}{lc}
\text { Operations \& } & \text { Network Switching } \\
\text { Maintenance } & \\
\text { System (OMC) } & \text { System (NSS) }
\end{array}
$$

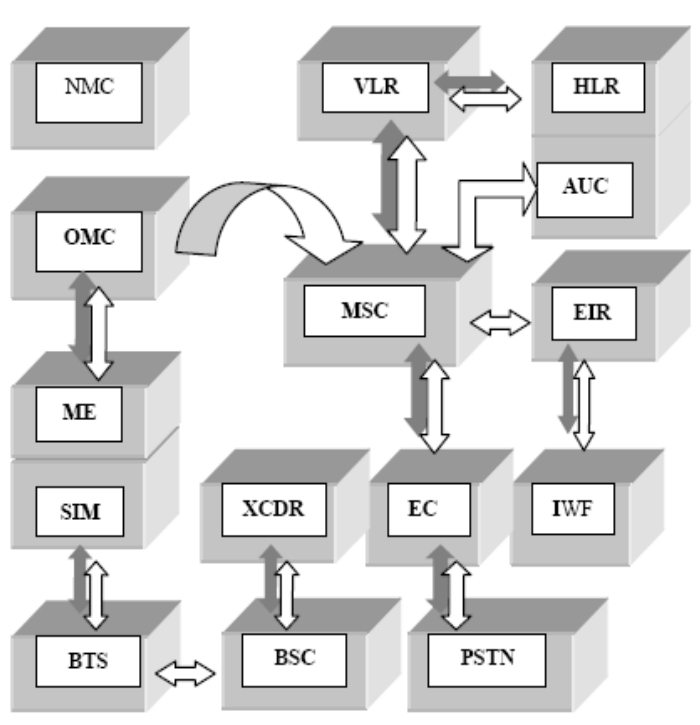

Figure 3. GSM System Architecture

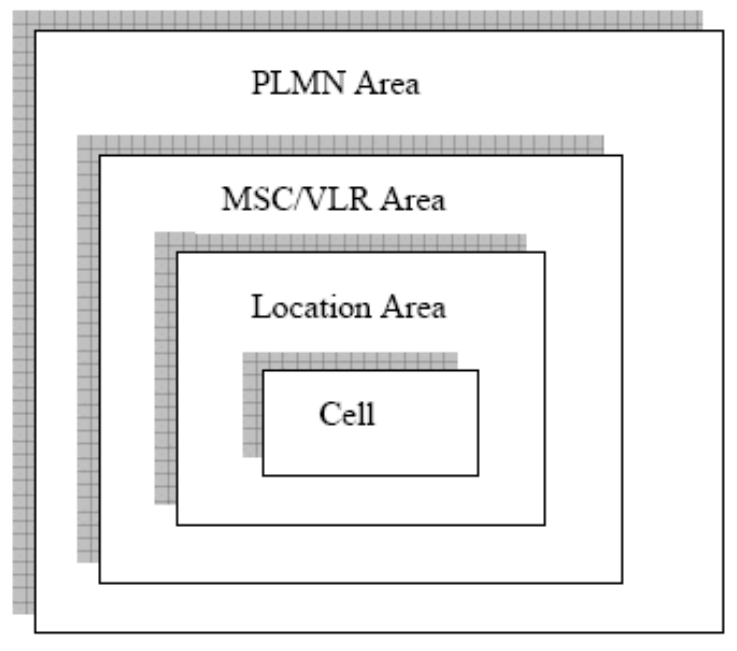

Figure 4. GSM Network Areas 


\section{SIGNAL POWER}

The selected area/zone for measurements is a multilevel geographical area/zone that needs a special solution for each case. Because an isotropic antenna has unity gain for both transmitter and receiver, hence the power received by such antenna is given by:

$$
\operatorname{Pr}=\operatorname{Pt}(\lambda / 4 \pi d)^{2}
$$

Where: Pr is the MS received power signal, $P t$ is the BS transmitted power signal, $d$ is the distance between the BS and the MS and $\lambda$ is the wavelength of the signal.

The above expression indicates that the attenuation is inversely proportional to the square of the distance between BS and MS [19] \& [20]. In practice of real system as illustrated in Figure 5 exist direct, diffracted and reflected paths. In this system a small difference between these three signals is mentioned. The received power can be written as:

$$
\operatorname{Pr}=\operatorname{Pt}(\lambda / 4 \pi d)^{2 *}\left|1+\rho e^{j \phi}\right|^{2}
$$

where $\rho$ is the reflection coefficient which is equal to -1 for an ideal reflector and $\phi$ is the phase difference between direct and reflected path.

Let the height of the BS and MS are HBS \&HMS respectively. The phase difference can be calculated using the following equation,

$$
\phi=4 \pi H_{B S} H_{M S} / \lambda d
$$

So, the received power can be written as,

$$
\operatorname{Pr}=\operatorname{Pt}(\lambda / 4 \pi d)^{2 *}\left|1-e^{j \phi}\right|^{2}
$$

Because the value of $\phi$ is very small, the second term is approximately equal to $\phi$

$$
\text { Therefore }\left|1-e^{j \phi}\right|=\phi
$$

Substituting equations $3 \& 5$ in equation 4 so

$$
\operatorname{Pr}=\operatorname{Pt}\left(H_{B S} H_{M S} / d^{2}\right)^{2}
$$

This equation is used in the frequency re-used calculation $[18] \&[20]$.

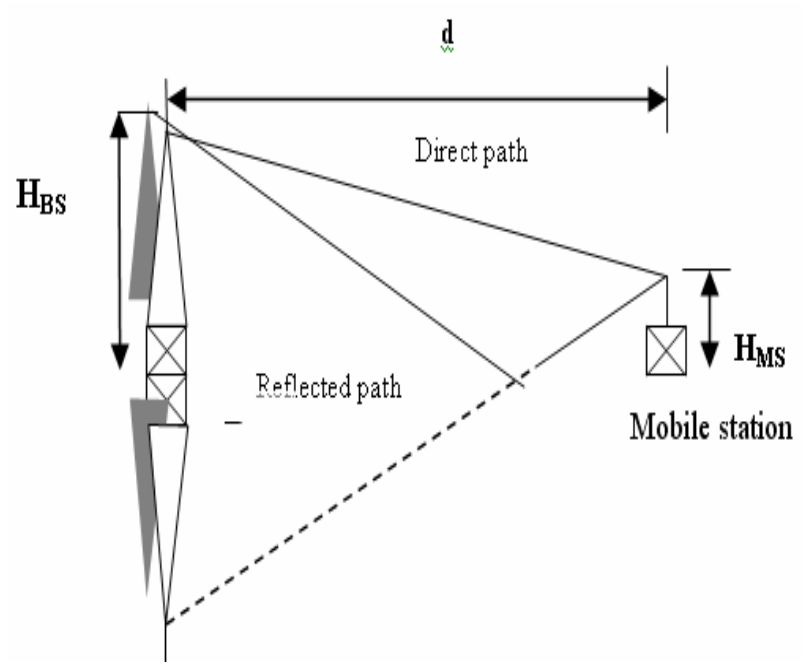

Figure 5. Multiple paths of GSM

\section{Proposed HANDOVER AlgorithM}

The subscribers' movement across the cells results in the need to change the allocated channels, which also happens when interference occurs. These events affect the quality of the communication so it is necessary to change the resources; defined as handover (HO) that is mainly controlled by the MSC.

The MS continuously monitors the strength of received signal strength either from its BS or the surrounded BSs, which depends on the list of all cells that must be monitored by its BS. This will help in deciding the destination of the MS, based on the best transmission of the surrounding cells in order to maintain the quality of the communication network.

The unwanted case (the worst case) situation occurs when the mobile is placed on the boundary of its serving cell. In this case the interference is produced by all overlapping surrounding cells. This case causes the strength and the quality of the signal to fall under a certain threshold. Therefore, HO must happen to maintain the call continuity.

In the GSM architecture shown in Figure (6) there are four possible types of $\mathrm{HO}$ which involve transferring a connection between two BSs:

- HO1: Channels in the same cell named Intra-cell handover

- HO2: Cells in the same BSC named Inter-cell, IntraBSC handover

- HO3: Cells in the different BSC named Inter BSC handover

- HO4: Intra MSC handover and cells in the different MSC named Inter MSC handover

Most cellular systems that employ GSM aim at maximizing HO for $60 \mathrm{~ms}$. The proposed algorithm depends on the continuous measurement at any point of the received power and the carrier to interference ratio. Depending of the refused values, a trade off process will be used to choose the best suitable value between these two measurements. The scanning process is necessary to check the mentioned values concerned with the received signal around all the nodes related to the MS. These processing, are used to verify various situations of handover problem, whenever it occurs during the crossing cells or MSC. When the problem is defined correctly, as indicated in the first phase, then the processing can be pass to the second phase that deals with the keeping continuity of the call during a correct decision.

The proposed algorithm is summarized by the following:

1. Management of received power measurements

2. Verification of the received power measurements

3. Management of carrier to interference ratio measurements

4. Verification of carrier to interference ratio measurements,

5. Comparison of measured and reference values

6. Selection of the optimal base station to connect the mobile

7. Handover decision depends on performance and the average value of the received signal 


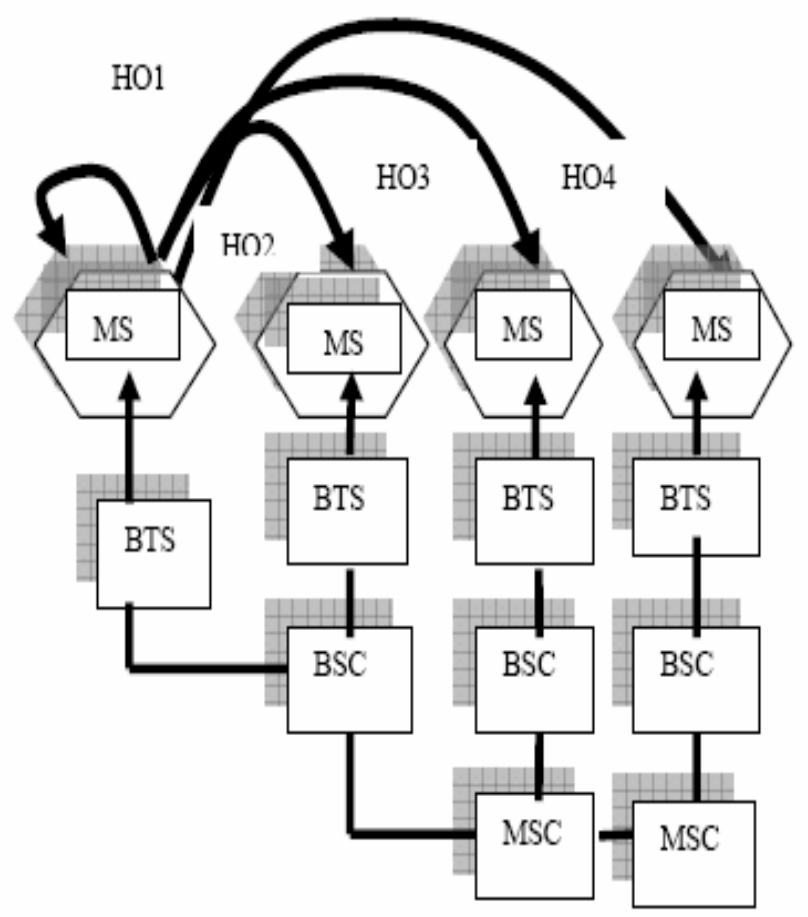

Figure 6. Possible types of handover

\section{SimULATION}

The Cellular systems require HO procedures to maintain the entire service area within an effective coverage range. The smaller cells require fast $\mathrm{HO}$ to recover calls within an adequate time and typical levels of the received signal, but this is different in large cells.

The behavior of the received signal level while a mobile device moves away from one BS to another seems to vary from one level to another. In this case, the hand over decision does not depend on the actual value of the received signal level, but on the average value.

The simulated results of the received power refers to the distance between the base station and the mobile station as demonstrated in Figure 7 (a) and (b) which are calculated at the same cell size. Graphs (c) and (d) in Figure 7 duplicate the cell size to determine its effects. Figure 8 illustrates the variation of carrier to interference ratio with respect to re-use frequency distance. These results are affected intensively by the topographic terrain.

\section{CONCLUSION}

The popularity of mobile phones and the number of mobile device users is continuously increasing, and at the same time mobile phone manufacturers are striving to introduce new feature-packed devices to hopefully attract potential new customers.

This work is limited and applied in a selected area that is situated in crowded noisy zones based on using GSM systems. This is due to the limitations that are already mentioned earlier in the introduction.

The presented HO algorithm is flexible and successful to select a proper significant path to take a correct decision. It also provides the ability to distinguish between real problems and false alarm problems. One of the important conclusions of the obtained results is that the pro-

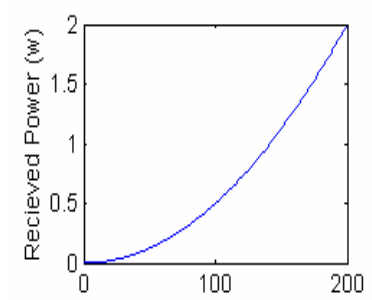

(a) Hight of the BS (m)

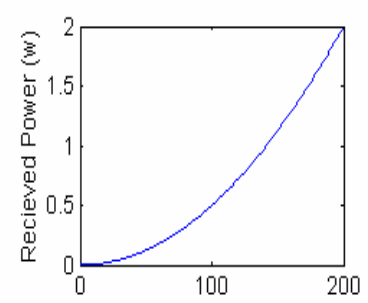

(b) Hight of the MS (m)
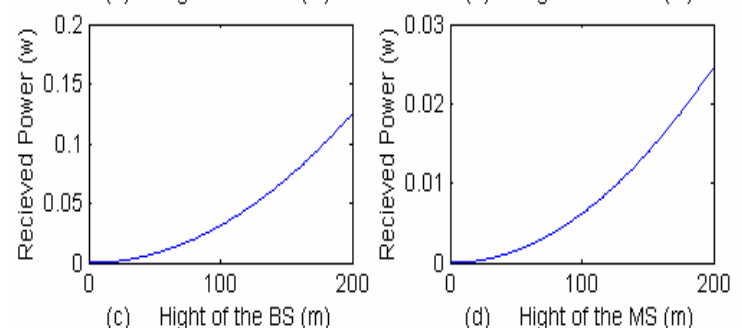

(d) Hight of the MS (m)

Figure 7. The received power with respect to the antenna height
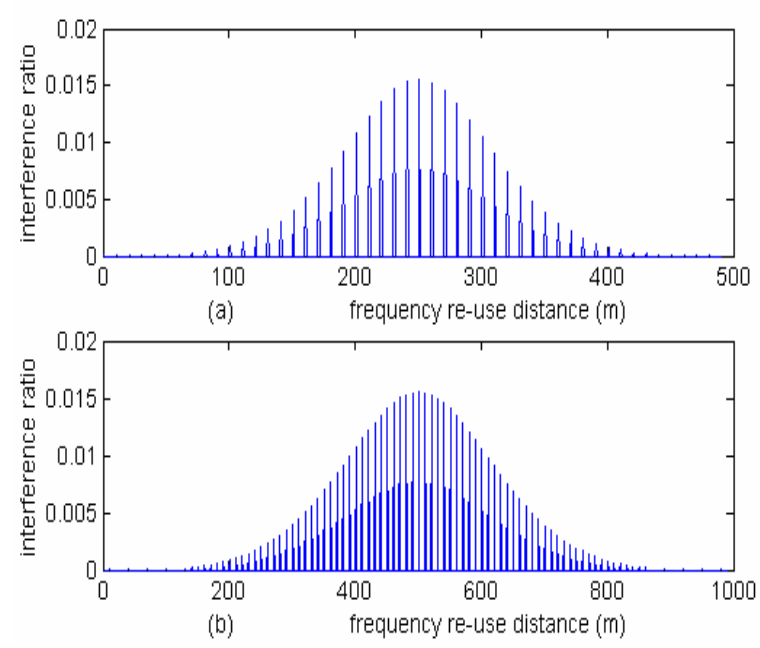

Figure 8. The received power with respect to the re-use frequency distance

proposed algorithm procedure maintains the ability to predict the adequate signal of the effective coverage cell.

More sophisticated handover mechanisms are needed for the transition from $2 \mathrm{G}$ networks to the more advanced mobile systems such as $3 \mathrm{G}$ networks, which are not available in all countries. The handover field offers a wide area for research and more challenges appear in the future when huge number of users access different types of wireless networks and different generations of mobile systems.

\section{ACKNOWLEDGMENT}

We would like to express our appreciations to Dr. Omar Daoud for his valuable assistance in proofreading this work.

\section{REFERENCES}

[1] A. Jagoda, M. Viillepin, and De Viillepin, "Mobile Communications", John Wiley and Sons, 1993.

[2] J.Dunlop and D.G. Smith, "Telecommunication Engineering", Prentice Hall, $3^{\text {rd }}$ edition, 1992.

[3] A. Alexiou, P.Kastarakis and V.N. Chand ristofilakis, "Interaction between GSM Handset Helical Antenna and User Head: Theoreti- 
cal Analysis Experimental Results", The Environmentalist, 25, 215-221, 2005. (doi:10.1007/s10669-005-4286-6)

[4] L. Rassian, "A Permutation Code Evolutionary Strategy for MultiObjective GSM Network Planning", J. Heuristics, 14,1-21, 2008. (doi:10.1007/s10732-007-9024-4)

[5] W. Scharnhorst et al., "Environmental Assessment of End-of-Life Treatment Options for A GSM 900 Antenna Rack", Int. J. LCA, 11, 6, pp.425-436, 2006. (doi:10.1065/lca2005.08.216)

[6] V.B. Manelis, I.V. Kaioukov, and A.V. Novikov, "Identification and Analysis of Interference Effects of GSM Base Stations", Radioelectronics and Communications Systems, vol.52, no.2, pp.5562, 2009. (doi:10.3103/S0735272709020010)

[7] L. Zhang etal., "A 1.8 Tri Mode $\sum \Delta$ Modulator for GSM/WCDMA/WLAN Wireless Receiver", Analog Integr Circ Sig Processing, 49, pp.423-441, 2006.

[8] K. Al-Begain et al., "Analysis of GSM/GPRS Cell with Multiple Data Service Classes", Wireless Personal Communication, 25, pp41-57,2003. (doi:10.1023/A:1023603308841)

[9] J. Tigang and F. Pingzhi, "Channel De-Allocation Schemes for GSM/GPRS Networks", Journal of Electronics (China), no.6, vol.22, 2005.

[10] J. Mar and J. Huang, "The Complementary Use of 3G WCDMA and GSM/GPRS Cellular Radio Networks", Wireless Personal Communications, 43, pp.511-531, 2007 (doi:10.1007/s11277-0079247-6)

[11] N. C. Lobely, "GSM to UMTS Architecture Evolution to Support Multimedia", B T Technol J, no.1, vol.19, January 2001.

[12] D. Obradovic and R. L. Scheiterer, "Troubleshooting in GSM Mobile Networks based on Domain Model and Sensory Information", ICANN LNCS, 3697, pp. 729-734, 2005

[13] M. E. Kounavis, and A. T. Campbell, "Design, Implementation and Evaluation of Programmable Handoff in Mobile Networks", Mobile Networks and Applications, Kluwer Academic Publishers, 2001.

[14] Pubudu N. Pathirana et al, "Mobility and Trajectory Prediction for Cellular Network with Mobile Base Station", $4^{\text {th }}$ international symposium on mobile Ad hoc networking and computing, 1-3, June 2003.

[15] M. E. Kounavis et al, "Supporting Programmable Handoff in Mobile Networks", $6^{\text {th }}$ International workshop on Mobile Multimedia Communications (MoMuC'99) San Diego, Ca, (November 1999).

[16] S. Seshan, et al, "Handoff in Cellular Network: the Daedalus Implementation and experience", Kluwer International Journal on Wireless Communication Systems, 1996.

[17] M. Dillenger and S. Buljore, "Reconfigurable Systems in Heterogeneous Environments", Software Defined Radio: Architectures, Systems and Functions, John Wiley and Sons, Ltd 2003.

[18] Payam Taaghol, "Optimization of WCDMA", Bechtel Telecommunication Technical Journal, Vol.2, No.1, 2004.

[19] S. Aust et al, "Policy Base Mobile IP Handoff Decision Using Generic Link Layer Information", Proceedings of the IEEE International Conference on Mobile and Wireless Communication Networks (MWCN 2003), Singapore, 27-29 October 2003.

[20] Gertie Alsenmyr et al, "Hand Over Between WCDMA and GSM "Ericson Review, No.1, 2003.

\section{AUTHORS}

Muzhir Al-Ani was born in Iraq in 1956. He received B. Sc. degree in Electrical Engineering from Sulaimania University, Iraq in 1979, Higher Diploma in Electronic and Communication Engineering from College of Engineering, University of Baghdad, Iraq in 1981, M. Sc. degree in Electronic and Communication Engineering from College of Engineering, University of Baghdad, Iraq in
1983. He received the Ph. D. degree in 1 July 1994 for his Thesis entitled Fast Algorithms of Digital Signal and Image Processing from Electronic Department of E.T.S.I.I. from University of Valladolid, Spain.

He joined in 7 July 1984 the Technical Institute of AlAnbar as an Assistant Lecturer. He worked as Assistant of the Dean in 1985, the Head of the Electrical Department during the years 1985 to 1988 .

He joined in 1994 the Electrical Engineering Department, College of Engineering, University of AlMustansiriya, Iraq, as a lecturer. He joined in 1996 Computer and Soft Engineering Department at the same College, working as the Head of Computer and Software Engineering Department during the years 1997 to 2001. In 5 May 1999 he was promoted Assistant professor (equivalent to Associate professor in Jordan) at the same Department. In 2 October 2001 he joined the Department of Computer Science and Information Systems in the University of Technology as the Head of the Department during the years 2001 to 2003 .

He joined in September 2003 Electrical Engineering and Computer Department Applied Science University, Amman, Jordan as Associate professor.

He joined in September 2005 Management Information System Department Applied Science University, Amman, Jordan as Associate professor, then in September 2008 Computer Science Department at the same University.

His research interests include Digital Signal Processing, Parallel Processing, Digital Filters, Digital Image Processing, Image Compression, Computer Vision, Information Systems, Information Hiding and Steganography, Wireless Networks Mobile Communications and related work.

(e-mail: muzhir@gmail.com)

Wael Hassan Al-Sawalmeh received Ph.D. degree in 1998 for his thesis entitled Working Out and Investigating of checking method of Color Production with help of sensors Based on LEDs and Fiber Optics of University of Telecommunication, Saint- Petersburg State-Russia.

He received M.Sc. degree in Electrical Engineering from Electro technical Institute of communication Leningrad, Bonch-Bruyevith, Russia in 1993.

He joined in 1998 university of Omar Al-Mouktar in Libya as assistant professor. In September 2001 he joined the Higher Institute of Comprehensive Vocational in AlBaida, Libya as a part time lecturer. In October 2001 he joined the Shahat Company for Computer Techniques in Al-Baida, Libya as a part time lecturer, in September 2002 he joined the Electrical Engineering and Computer Department Applied Science University, Amman, Jordan as Assistant professor. He joined in September 2004 Communication \& Electronics Department Philadelphia University, Amman, Jordan as Assistant professor.

His research interests include TV, mobile communications and Digital Signal Processing.

(e-mail: waelalsawalme@hotmail.com)

Submitted 17 February 2009. Published as resubmitted by the authors on 9 October 2009 